\title{
Extension of Branched Chain of Amylopectin by Enzymatic Reaction and its Structural Characterization
}

(Received December 26, 2012; Accepted February 28, 2013)

(J-STAGE Advance Published Date: March 11, 2013)

\author{
Yoshiaki Yuguchi, ${ }^{1, *}$ Kenji Hashimoto, ${ }^{1}$ Kyoko Yamamoto, ${ }^{1}$ Shiho Suzuki ${ }^{2}$ and Shinichi Kitamura ${ }^{2}$ \\ ${ }^{1}$ Faculty of Engineering, Osaka Electro-Communication University \\ (18-8 Hatsu-cho, Neyagawa, Osaka 572-8530, Japan) \\ ${ }^{2}$ College of Life, Environment, and Advanced Sciences, Osaka Prefecture University \\ (1-1 Gakuen-cho, Sakai, Osaka 599-8531, Japan)
}

\begin{abstract}
Amylose can be synthesized with phosphorylase (EC 2.4.1.1) by using malto-oligosaccharides as primers. In this context, the glucose-1-phosphate (G-1-P) units are consumed through the polymerization process. In this study, we investigated the use of amylopectin as a primer for the synthesis of a novel starch. The obtained starch was characterized by dynamic light scattering, small-angle X-ray scattering (SAXS) and X-ray diffraction. The SAXS results were well represented by the star-polymer model scattering function. According to these results, we think that the amylopectin chain elongation was implied, and the transformation of crysralline type of amylopectin was observed by X-ray diffraction.
\end{abstract}

Key words: amylose, amylopectin, phosphorylase, small-angle X-ray scattering

\section{INTRODUCTION}

Starch is a mixture of amylose, a linear polysaccharide composed of $\alpha-1,4$-linked glucose units, and amylopectin, constituted by an amylose-like skeleton with occasional $\alpha-1,6$-glycosidic bonds which provide branching points. As the content ratio of amylose and amylopectin and their structural characteristics affect the properties of food, many studies have focused, for a long time, on the structural analysis of starch. Recently, starch has attracted much attention for its possible employment in the development of bio-fuels and bio-plastics. Therefore, structural information on starch and the applications of the phosphorylase enzyme are very important subjects.

Amylose chain coils into a helical shape, and amylopectin has a specific fringed arrangement with the branched chains, forming double helical structures. The types of structures of amylopectin are classified as A-type from cereals like corn, and B-type from roots like potatoes. ${ }^{1)}$ The formation of a crystal structure from a starch solution has been discussed in terms of dependence on concentration and/or molecular weight. ${ }^{2)}$ A crystal cluster generates a peak in the small-angle X-ray scattering (SAXS) profile. ${ }^{3-5)}$ This behavior may be due to the correlation hole induced from the periodic density inhomogeneity of 2 regions of cluster and amorphous structure. Such an assembly yields the starch granule and confers the starch its characteristic hierarchical structure.

In plants, various types of enzymes are responsible for producing starches with the appropriate structure and energy

\footnotetext{
*Corresponding author (Tel. +81-72-824-1131, Fax. +81-72-824-0014, E-mail: yuguchi@isc.osakac.ac.jp).

Abbreviations: G-1-P, Glucose-1-phosphate; SAXS, Small-angle Xray scattering; SEC-MALLS, size exclusion chromatography-multiangle laser light scattering.
}

storage abilities. By exploiting these enzymes, it is possible to design specific molecular structures and to create novel functional biomaterials. Amylose can be synthesized with phosphorylase (EC 2.4.1.1) by using malto-oligosaccharides as primers. The glucose-1-phosphate (G-1-P) can be polymerized by exploiting the catalytic properties of phosphorylase. ${ }^{6}$ Gelders et al. have reported on the potato phosphorylase-catalyzed synthesis of amylose in the presence of lipids. ${ }^{7)}$ In that report, amylose was seen to form a complex with the lipids and to complete its polymerization by precipitation. The molecular weight of the obtained amylose depended on the length of the hydrocarbon chain of the lipid. Such findings implied that the actual molecular weight of amylose could be controlled. Putaux et al. prepared dendritic nanoparticles by enzymatic chain extension of glycogen ${ }^{8)}$ by using amylosucrase in the presence of sucrose as the glucose donor. The diameter of those particles was 4-5 times bigger than the initial glycogen, but shrank after partial crystallization. Izawa et al. discovered a novel gelation system by exploiting the phosphorylase-catalyzed chain elongation of glycogen. ${ }^{9)}$ The glycogen particles were linked to each other and formed a helix between the elongated amylose chains.

In this study, we used the same enzymatic synthesis strategy to induce branched chain extensions in amylopectin. SAXS experiments were employed to characterize the chain architecture of the novel synthesized starches.

\section{MATERIALS AND METHODS}

Extraction of phosphorylase. We modified the potato phosphorylase purification method conceived by Kamogawa et al. ${ }^{10)}$

The purification process was carried out under ice cooling 
conditions. Mashed potatoes (100 g) were filtered with a cloth and the resulting filtrate was centrifuged at 4,000 rpm for $20 \mathrm{~min}$. The supernatant was incubated with $0.5 \mathrm{~g}$ of DEAE Sephadex A50 beads for $30 \mathrm{~min}$. Sodium chloride was added to the gel dispersion to a final concentration of $1.5 \%$. Then, the gel was filtered and incubated for $20 \mathrm{~min}$ in a $3.5 \% \mathrm{NaCl}$ aqueous solution. This dispersion was filtered with filter paper, and the filtrate containing the phosphorylase was stored in the refrigerator until use. The phosphorylase activity was estimated as $29 \mathrm{U} / \mathrm{mL}$ by quantitative analysis of free phosphate in the reaction mixture.

Sample preparation. Maltopentaose and Lintner's soluble waxy corn starch (Hayashibara Co. Ltd., Okayama, Japan) were used as primers. G-1-P was provided by Wako Pure Chemical Industries, Ltd. (Osaka, Japan).

The mixture of primer and G-1-P in $0.2 \mathrm{M}$ acetate buffer (pH 5.9) was prepared and the reaction was started by adding $10 \% \mathrm{v} / \mathrm{v}$ of the stored phosphorylase solution. The reaction was run at $50^{\circ} \mathrm{C}$ for $42 \mathrm{~h}$, and then stopped by increasing the temperature to $100^{\circ} \mathrm{C}$. The product was obtained by precipitation with ethanol, filtered with a $0.2 \mu \mathrm{m}$ membrane filter, and dried to yield a solid sample. Several samples were synthesized by using different weight fractions of primer to G-1-P, ranging from 0.01 to 0.2 .

SEC-MALLS. The average molecular weights ( $M$ ws $)$ and molecular weight distributions $\left(M_{\mathrm{w}} / M_{\mathrm{n}}\right)$ of the synthesized products were determined by size exclusion chromatography-multi-angle laser light scattering (SEC-MALLS) measurements conducted on an HPLC system. The HPLC instrument was equipped with 3 connected columns: TSKgel G6000PWXL, G5000PWXL and G4000PWXL (7.5 mm i.d. $\times 600 \mathrm{~mm}, 25^{\circ} \mathrm{C}$; Tosoh Corporation, Tokyo, Japan). The column outlet was connected to a Dawn Heleos-II multiangle laser light scattering photometer (Wyatt Technology Corp., Goleta, USA) $(\lambda=658 \mathrm{~nm})$ which was, in turn, connected to an Optilab rEX differential refractometer (Wyatt Technology Corp.). The mobile phase was $0.2 \mathrm{M}$ $\mathrm{KNO}_{3}$, and the flow rate was $0.7 \mathrm{~mL} / \mathrm{min}$. The injection volume was $100 \mu \mathrm{L}$, with the sample concentration varying from 0.4 to $1.0 \%$ for securing an appropriate light scattering intensity. The samples were heated at $135^{\circ} \mathrm{C}$ in a pressuretight reactor, and the resultant solution was filtered with a $0.45 \mu \mathrm{m}$ filter before injection. A refractive index increment $(\delta n / \delta c)$ of $0.150 \mathrm{~mL} / \mathrm{g}$ was used for amylose. Data from the light scattering and the differential refractometer were collected and processed using Astra (v. 5.3.4.14) software (Wyatt Technology Corp.).

Dynamic light scattering. The dynamic light scattering was measured with Autosizer 4700C (Malvern Instruments, Ltd., Malvern, UK). The samples have been irradiated with a He-Ne laser $(\lambda=633 \mathrm{~nm})$, and the scattered light was detected at $90^{\circ}$. The sample solutions were constituted by $0.4 \%$ polymers in $1 \mathrm{M} \mathrm{KOH}$.

Small-angle X-ray scattering. The SAXS experiments were performed at BL40B2 in the SPring-8, Hyogo, Japan. The incident X-ray beam adjusted to $0.1 \mathrm{~nm}$ was used to irradiate the sample cells, after which the scattered X-rays were detected with Imaging Plate (IP). The 2-dimensional data thus acquired were circularly averaged to transform into 1-dimensional data. The excess scattering intensities were calculated by subtracting the scattering intensities of solvent.
X-ray diffraction. X-ray diffraction patterns were taken with an X-ray diffractometer (Rigakudenki RINT Ultima+, Rigaku Corporation, Tokyo, Japan) using the following conditions: X-ray $(\mathrm{CuK} \alpha)$ wavelength, $0.154 \mathrm{~nm}$; scanning step, $0.020^{\circ}$; time constant, $1 \mathrm{~s}$. The powder samples were used in a dried state without humidity control.

\section{RESULTS AND DISCUSSION}

Lintner's soluble waxy corn starch and maltopentaose were used as primers to produce enzymatically synthesized amylose and novel amylopectin with elongated chains, respectively. The different weight fractions of primers against G-1-P tested in the synthesis are summarized in Table 1, which also indicates the sample codes. The distribution of the molecular weights of the starches was analyzed by SEC-MALLS (see Table 1). In the MP series, which comprise the starches synthesized with maltopentaose as primer, the molecular weights tended to decrease when the weight fraction of the primer increased. Considering the amount of G-1-P consumed for each primer, this is a reasonable behavior. Since Lintner's soluble waxy corn starch is produced by acidic hydrolysis of waxy corn starch, it is soluble in water when heated to approximately $80^{\circ} \mathrm{C}$. Therefore, the enzymatic reaction is started with a solvated primer. The molecular weight of Lintner's soluble waxy corn starch is widely distributed, with an average value of $1.63 \times$ $10^{5} \mathrm{Da}$. The evaluated molecular weights in the AP series depended on the weight fraction of primer with the same relationship as the MP series.

As shown in Fig. 1, the size of each starch molecule solvated in $1 \mathrm{M} \mathrm{KOH}$ solution was observed by dynamic light scattering. The starch is insoluble in water because of its strongly stabilized crystal structure due to hydrogen bonding and helix formation, which is why the samples were solvated with an alkaline solution of $\mathrm{KOH}$. The sizes of linear amylose samples (MP series) are distributed within the range of 200-300 nm, and the dimensions increased with increasing molecular weight. The Lintner's soluble waxy corn starch was elongated and extended from its original size of $22 \mathrm{~nm}$ to sizes ranging from 25 to $35 \mathrm{~nm}$. The size of the AP series also increased with an increase in the molecular weight, even though it was much smaller than that of the MP series because of the branched structure.

Table 1. The weight faction of primer for each samples and the molecular weight of them evaluated by SEC-MALLS.

\begin{tabular}{ccccc}
\hline \multirow{2}{*}{ Primer } & Sample code & $\begin{array}{c}\text { Weight } \\
\text { fraction of } \\
\text { primer for } \\
\text { G-1-P }\end{array}$ & $\begin{array}{c}\text { Molecular } \\
\text { weight } \\
(M \mathrm{w}) \times 10^{-5}\end{array}$ & Mw/Mn \\
\hline \multirow{2}{*}{ Maltopentaose } & MP 0.01 & 0.01 & 0.17 & 1.02 \\
& MP 0.02 & 0.02 & 0.11 & 1.01 \\
& Lintner's & 0.10 & 0.05 & 1.02 \\
\hline Lintner's & solublewaxy & 0.00 & 1.63 & 4.78 \\
coluble waxy starch & & & \\
\cline { 2 - 5 } corn starch & AP 0.01 & 0.01 & 2.45 & 3.81 \\
(Amylopectin) & AP 0.02 & 0.02 & 2.45 & 4.55 \\
& AP 0.1 & 0.10 & 2.31 & 3.30 \\
& AP 0.2 & 0.20 & 2.17 & 2.99 \\
\hline
\end{tabular}



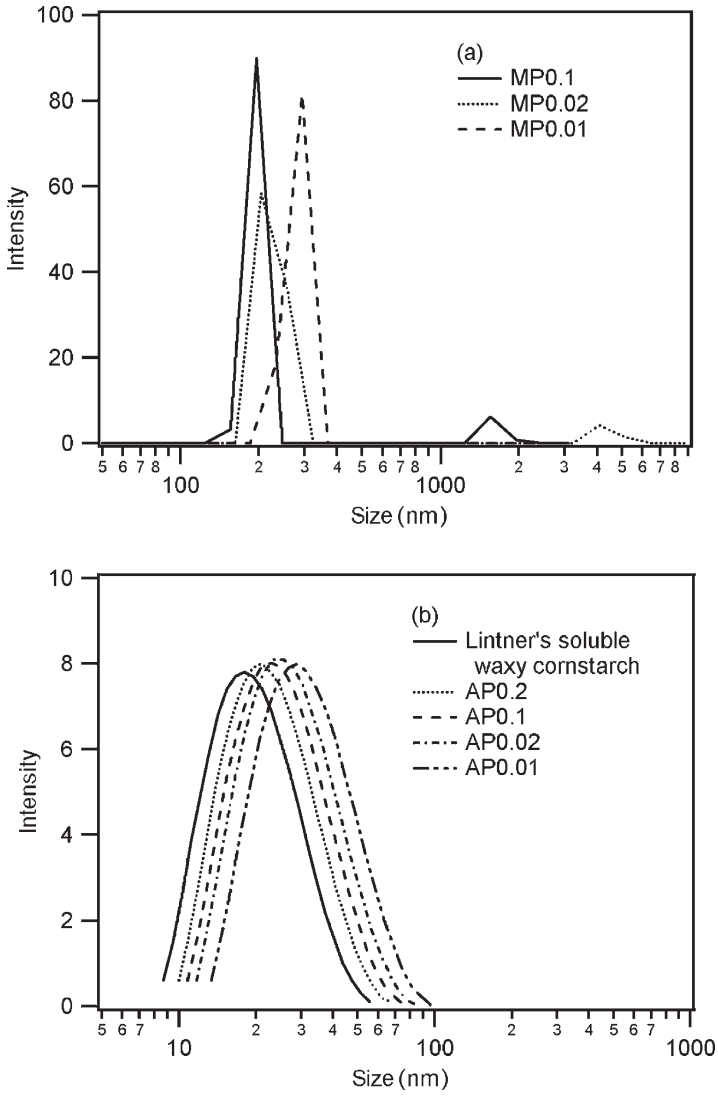

Fig. 1. Particle size distribution of the obtained starches evaluated by dynamic light scattering.

The SAXS method was used to observe the local conformations of the obtained starches. Figure 2 represents the Kratky plots $\left(q^{2} I(q) \text { vs. } q\right)^{11)}$ for small-angle scatterings (SAS) of primers and enzymatically synthesized starches in $1 \mathrm{M} \mathrm{KOH}$ solutions. $q$ is the magnitude of the scattering vector and $I(q)$ is the scattering intensity. Here $q$ is defined by $(4 \pi / \lambda) \sin \theta$, where $\lambda$ is the wavelength of the incident $\mathrm{X}$-ray and $2 \theta$ is the scattering angle. As shown in Fig. 2 (a) the X-ray scattering from synthesized amylose has a maximum around $2 \mathrm{~nm}^{-1}$ of $q$, which does not originate from the maltopentaose primer. The maximum in the scattering profiles is assigned to the amylose chain elongation. The result is consistent with the conclusion that the chain grew long enough to form a helical structure. The maximum mainly reflects the scattering from the cross-section of the rod-like structure. Since amylose tends to coil into a helix, the single helix model could be adopted (Fig. 3). The scattering from the molecular model can be calculated with the Debye formula ${ }^{12)}$ as

$$
\begin{aligned}
I(q)= & \sum_{j=1}^{n} f_{i}^{2} g_{j}^{2}(q)+2 \sum_{j=1}^{n-1} \sum_{k=j+1}^{n} f_{j} f_{k} g_{j}(q) g_{k}(q) \\
& \times \frac{\sin d_{j k} q}{d_{j k} q}
\end{aligned}
$$
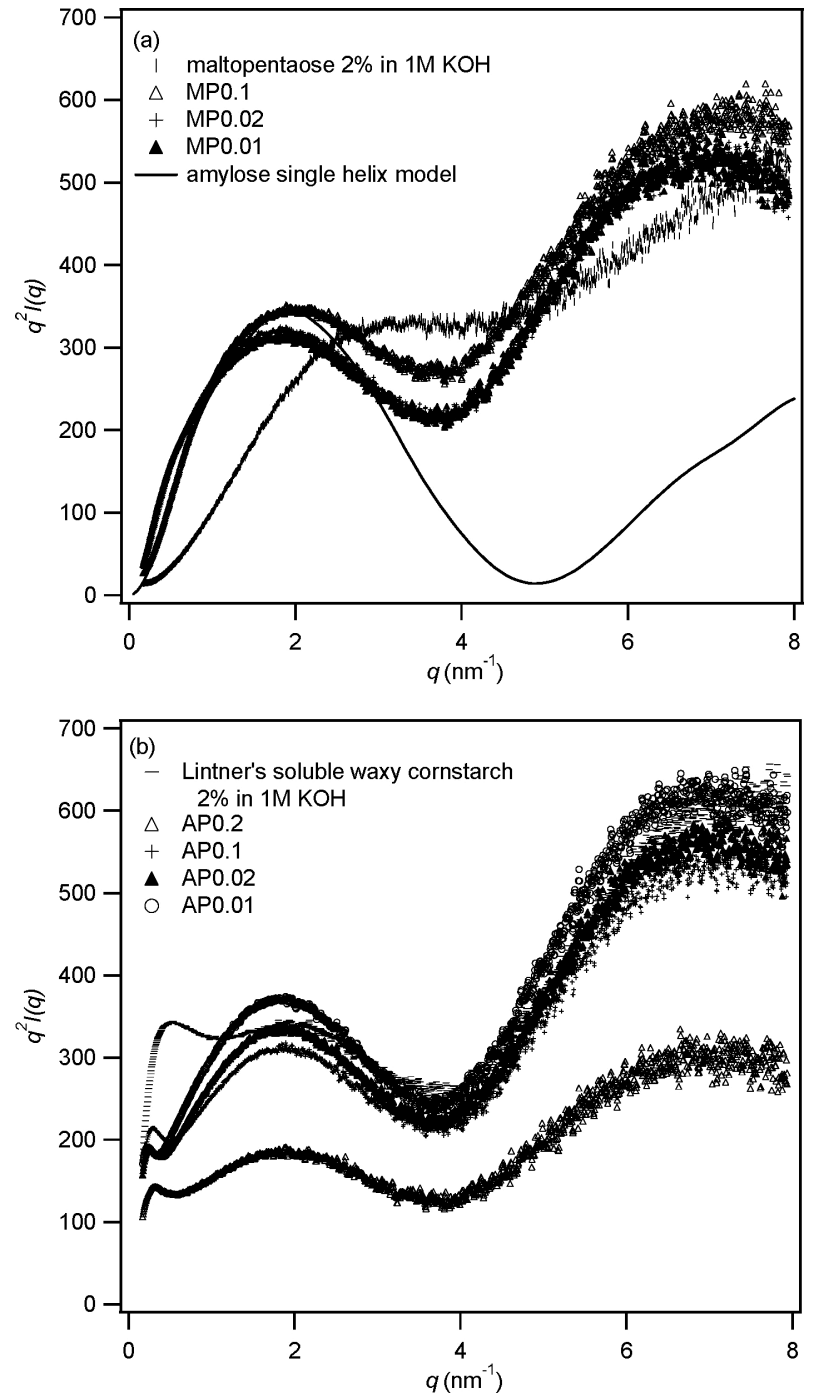

Fig. 2. Kratky plots $\left(q^{2} I(q)\right.$ vs. $\left.q\right)$ for small angle X-ray scattering (SAXS) from the primers and enzymatically synthesized starches in $1 \mathrm{M} \mathrm{KOH}$ solutions.

(a) MP series, (b) AP series.

where $f_{i}$ and $d_{j k}$ are the atomic scattering weight of the atom $j$ and the distance between the $j^{\text {th }}$ and $k^{\text {th }}$ atoms, respectively. The form factor $g_{j}(q)$ for a single atom is represented by a rigid sphere with a radius equal to the van der Waals radius $(R j)$ of the $j^{\text {th }}$ atom.

$$
g_{j}=\frac{3\left[\sin \left(R_{j} q\right)-\left(R_{j} q\right) \cos \left(R_{j} q\right)\right]}{\left(R_{j} q\right)^{3}}
$$

As shown in Fig. 2 (a), the position of the maximum of the simulated curve is consistent with the experimental profile. This finding shows that the amylose chain partially retains its helical structure even in solution. In the AP series, differently from the MP series, the peak appeared in the region of smaller angles. Therefore, we attributed the peak to the branched architecture of amylopectin. In fact, the chain

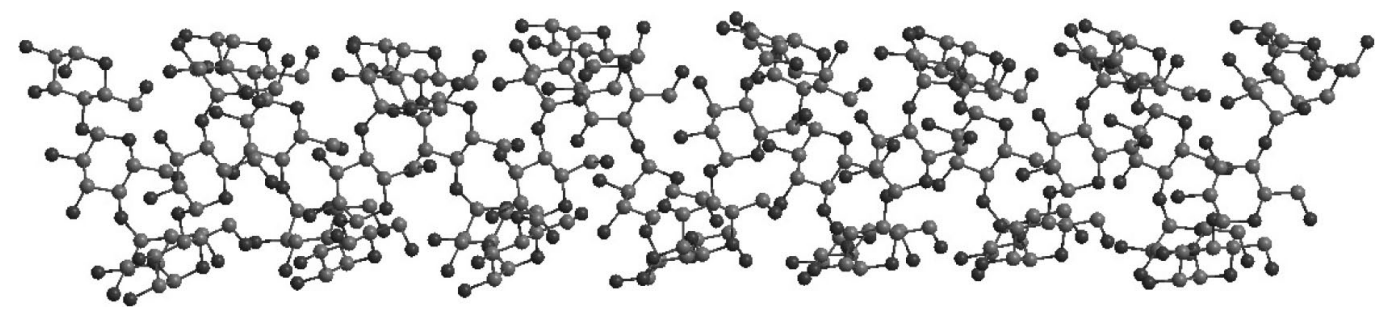

Fig. 3. Molecular model of a single amylose helical chain. 


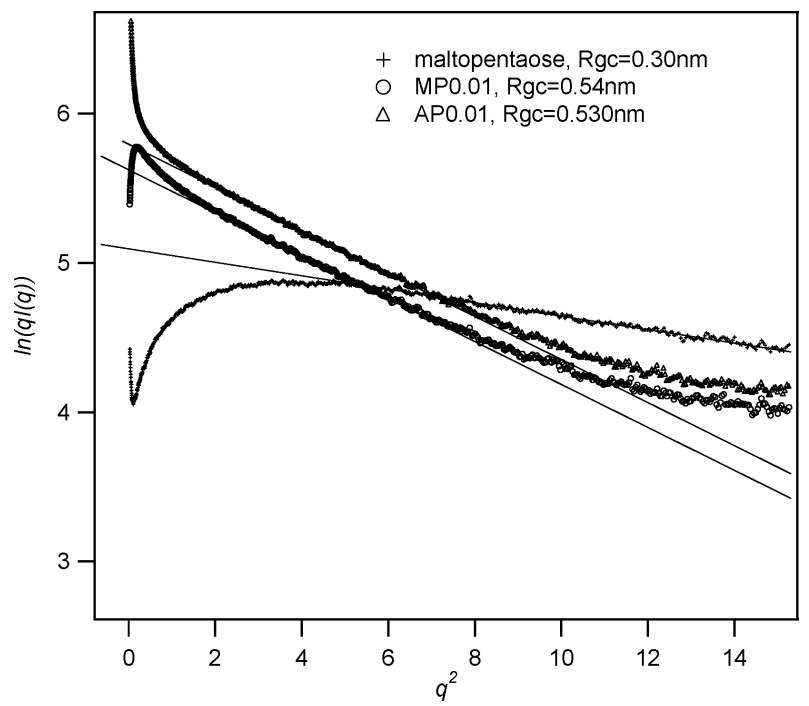

Fig. 4. Cross-sectional Guinier plots of SAXS of maltopentaose, MP0.01 and AP0.01.

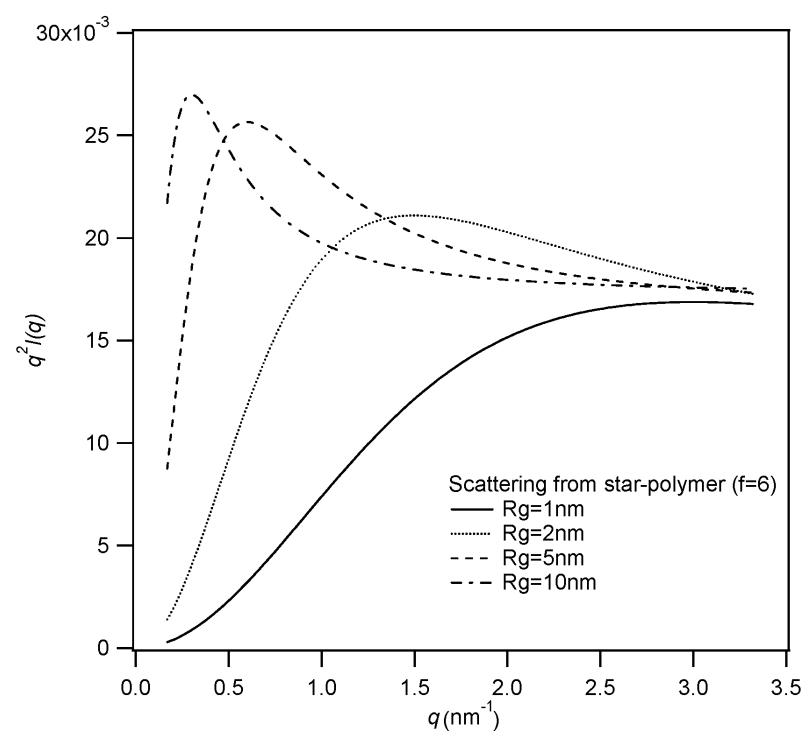

Fig. 5. Kratky plots for simulated SAXS of star polymers with different radii of gyration.

extension promoted by the enzymatic reaction with the Lintner's soluble waxy corn starch induced a shift to a smaller angle value $\left(5 \mathrm{~nm}^{-1}\right)$. However, the scattering profile was similar to that of the wider-angle region in MP series. This result indicates that the local chain structure was the same as in the linear amylose in solution.

The scattering from rod-like particles can be given by the Guinier approximation as

$$
q \cdot I(q) \approx \exp \left(-q^{2} R g, c^{2} / 2\right)
$$

where $R g, c$ is the cross-sectional radius of gyration. $R g, c$ can be evaluated from the slope in the cross-sectional Guinier plots $\left(\ln (q I(q)) v s . q^{2}\right)^{13-14)}$ in the appropriate region. Figure 4 depicts the cross-sectional Guinier plots of the SAXS measurements of maltopentaose, MP0.01 and AP0.01. While the $R g, c$ value of maltopentaose is $0.3 \mathrm{~nm}, R g, c$ values for the reacted samples were estimated at approximately $0.5 \mathrm{~nm}$, corresponding to the cross-section of a helical structure. In fact, the smaller $R g, c$ value of maltopentaose because it cannot adopt a helical structure, the reason being its short chain length.

To explain the scattering behavior of the branched chains,

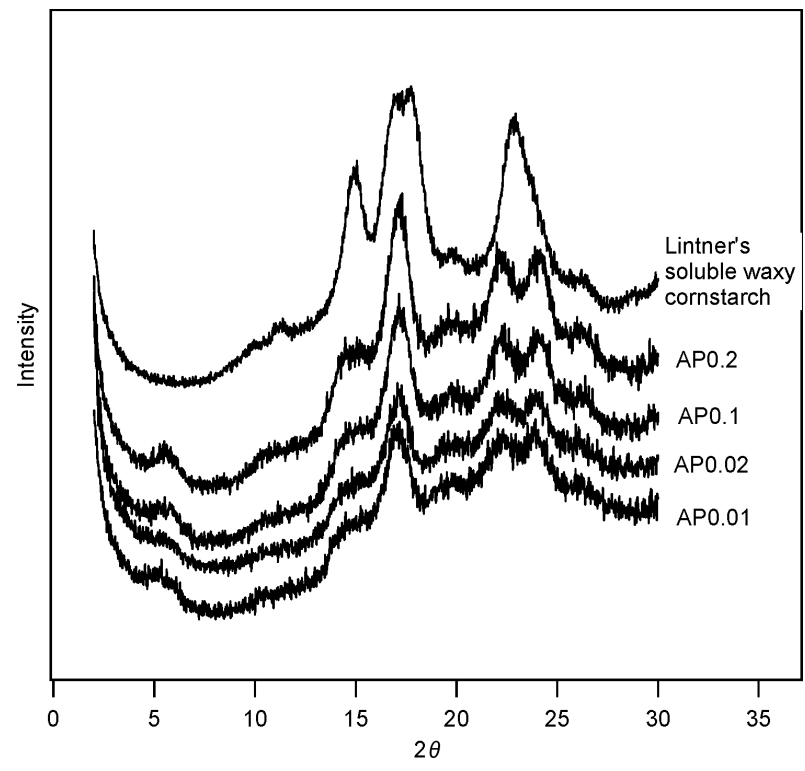

Fig. 6. X-ray diffraction patterns from Lintner's soluble waxy corn starch and AP series.

we simulated their scattering profiles with the star polymer scattering function, which is described as

$$
I(q) \approx \frac{1+\operatorname{Rg}^{2} q^{2} / 3 f}{\left[1+\operatorname{Rg}^{2} q^{2}(f+1) / 3 f\right]^{2}}
$$

where $f$ is the degree of the branch, and $R g$ is the radius of gyration of the star polymer. ${ }^{15-16)}$ The poly-disperse rays of the star are characterized by the Schultz-Flory distribution. Figure 5 represents the Kratky plots for the simulated SAXS of star polymers with different radii of gyration. When the $R g$ value increases, the branched chain length is extended and the peak position shifts to smaller angle values. Because the experimental data have the same tendency, the star polymer scattering function provides a good simulation for the local branched structures. Here, the values of $f=6$ and $R g$ $=1-10 \mathrm{~nm}$ were used. In this experiment, the real structure of amylopectin was very complex, and the scattering profile was averaged over different amylopectin forms and structures. Therefore, it is unlikely that the parameters that have been used properly represent the real molecules. Nonetheless, the simulation of the data is still very important to reveal the tendency of the scattering behavior.

Figure 6 shows the powder X-ray diffraction patterns of Lintner's soluble waxy corn starch and AP series. The pattern of the original reagent indicates an A-type crystalline structure that is transformed into a B-type one by the enzymatic reaction and chain elongation. Generally, longer branched chains tend to originate B-type crystalline granules, because of which our result also supports the chain extension model. ${ }^{17)}$

\section{ACKNOWLEDGMENTS}

We thank the Japan Synchrotron Radiation Research Institute (JASRI) (Proposal No. 2011B1277, No. 2012A1261) for approving the use of their facilities. We are also thankful for the financial support from the Osaka Electro-Communication University.

\section{REFERENCES}

1 ) A. Imberty, A. Buléon, V. Tran and S. Pérez: Recent advances in knowledge of starch structure. Starch/Stärke, 43, 375-384 (1991). 
2 ) M.J. Gidly and P.V. Bulpin: Crystallisation of malto-oligosaccharides as models of the crystalline forms of starch: minimum chain-length requirement for the formation of double helices. Carbohydr. Res., 161, 291-300 (1987)

3 ) S. Pikus: Small-angle X-ray scattering (SAXS) studies of the structure of starch and starch products. Fibers Text. East. Eur., 13, 82-86 (2005)

4 ) A.M. Donald, P.A. Perry and T.A. Waigh: The impact of internal granule structure on processing and properties. in Starch: Advanced in Structure and Function, T.L. Barsby, A.M. Donald and P.J. Frazier, eds., Royal Society of Chemistry, London, pp. 45-52 (2001)

5 ) S.V. Gomand, L. Lamberts, C.J. Gommes, R.G.F. Visser, J.A. Delcour and B. Goderis: Molecular and morphological aspects of annealing-induced stabilization of starch crystallites. Biomacromology, 13, 1361-1370 (2012).

6 ) M.J. Gidley and P.V. Bulpin: Aggregation of amylose in aqueous system: the effect of chain length on phase behavior and aggregation kinetics. Macromology, 22, 341-346 (1989).

7 ) G.G. Gelders, H. Goesaert and J.A. Delcour: Potato phosphorylase catalyzed synthesis of amylose-lipid complexes. Biomacromology, 6, 2622-2629 (2005).

8 ) J.L. Putaux, G.P. Véronèse, M.R. Simeon and A. Buleon: $\alpha$-D-glucam based dendric nanoparticles prepared by in vitro enzymatic chain extension of glycogen. Biomacromology, 7, 1720-1728 (2006)

9 ) H. Izawa, M. Nawaji, Y. Kaneko and J. Kadokawa: Preparation of glycogen-based polysaccharide materials by phosphorylasecatalyzed chain elongation of glycogen. Macromol. Biosci., 9, 1098-1104 (2009).

10) A. Kamogawa, T. Fukui and Z. Nikuni: Potato $\alpha$-glucan phosphorylase: crystallization, amino acid composition and enzymatic reaction in the absence of added primer. J. Biochem., 63, 361-369 (1968).

11) O. Kratky: Natural high polymers in the dissolved and solid state. in Small Angle X-ray Scattering, O. Glatter and O. Kratky, eds., Academic Press, London, pp. 361-386 (1982).

12) P. Debye: Scattering from non-crystalline substances. Ann. Physik, 46, 809-823 (1915).

13) Y. Yuguchi, T.T.T. Thuy, H. Urakawa and K. Kajiwara: Structural characteristics of carrageenan gels: temperature and concentration dependence. Food Hydrocoll., 16, 515-522 (2002).

14) Y. Yuguchi, T. Hirotsu and J. Hosokawa: Structural characteristics of xyloglucan - congo red aggregates as observed by small angle X-ray scattering. Cellulose, 12, 469-477 (2005)

15) W. Burchard: Statistics of star-shaped molecules. II. Stars with homodisperse side chains. Macromology, 7, 841-846 (1974).

16) W. Burchard: Particle scattering factors of some branched polymers. Macromology, 10, 919-927 (1977).

17) A. Kubo, Y. Yuguchi, M. Takemasa, S. Suzuki, H. Satoh and S. Kitamura: The use of micro-beam X-ray diffraction for the characterization of starch crystal structure in rice mutant kernels of waxy, amylose extender, and sugaryl. J. Cereal Sci., 48, 92-97 (2008). 